\title{
Large Space Optics: from Hubble to JWST and Beyond
}

\author{
H. Philip Stahl, Ph.D. \\ NASA Marshall Space Flight Center \\ Huntsville, $A L 25812$ \\ h.philip.stahl@nasa.gov
}

\begin{abstract}
Evolution of large space optics from medium weight monolithic egg-crate mirrors for Hubble to lightweight segmented mirrors for JWST to future architectures ranging from ultralightweight membrane mirrors to massive mirrors launched via planned Ares V.

OCIS codes: (350.1260) Astronomical optics; (350.4600) Optical engineering; (220.4610) Optical fabrication; (220.4830) Systems design
\end{abstract}

\section{Launch Vehicle Constraints have driven Space Mirror Technology}

If necessity truly is the mother of invention, then advances in lightweight space mirror technology have been driven by launch vehicle mass and volume constraints. In the late 1970's, at the start of Hubble development, the state of the art in ground based telescopes was 3 to 4 meter monolithic primary mirrors with masses of 6000 to 10,000 kg clearly too massive for the planned space shuttle 25,000 kg capability to LEO. 'Necessity' led Hubble to a different solution. Launch vehicle mass constraints (and cost) resulted in the development of a 2.4 meter lightweight eggcrate mirror. At $810 \mathrm{~kg}(180 \mathrm{~kg} / \mathrm{m} 2)$, this mirror was approximately $7.4 \%$ of HST's total 11,110 kg mass. And, the total observatory structure at $4.3 \mathrm{~m}$ x $13.2 \mathrm{~m}$ fit snuggly inside the space shuttle $4.6 \mathrm{~m} \times 18.3 \mathrm{~m}$ payload bay.

In the early 1990's, at the start of JWST development, the state of the art in ground based telescopes was 8 meter class monolithic primary mirrors (16,000 to 23,000 kg) and 10 meter segmented mirrors (14,400 kg). Unfortunately, launch vehicles were still constrained to 4.5 meter payloads and 25,000 $\mathrm{kg}$ to LEO or 6,600 $\mathrm{kg}$ to L2. Furthermore, science now demanded a space telescope with 6 to 8 meter aperture operating at L2. Mirror technology was identified as a critical capability necessary to enable the next generation of large aperture space telescopes. Specific telescope architectures were explored via three independent design concept studies conducted during the summer of 1996 (1). These studies identified two significant architectural constraints: segmentation and areal density. Because the launch vehicle fairing payload dynamic envelop diameter is approximately 4.5 meters, the only way to launch an 8 meter class mirror is to segment it, fold it and deploy it on orbit - resulting in actuation and control requirements. And, because of launch vehicle mass limits, the primary mirror allocation was only $1000 \mathrm{~kg}-$ resulting in a maximum areal density of $20 \mathrm{~kg} / \mathrm{m} 2$. At the inception of JWST in 1996, such a capability did not exist. A highly successful technology development program was initiated resulting in matured and demonstrated mirror technology for JWST $(2,3)$. Today, the JWST 6.5 meter primary mirror has an areal density of $25 \mathrm{~kg} / \mathrm{m} 2$ for a total mass of $625 \mathrm{~kg}$ or $9.6 \%$ of the total JWST observatory mass of $6,500 \mathrm{~kg}$.

Looking into the future, science requires increasing larger collecting apertures. Ground based telescopes are already moving towards $30+$ meter mirrors. The only way to meet this challenge for space telescopes is via even lower areal density mirrors or on-orbit assembly or larger launch vehicles (4). The planned NASA Ares V with its 10 meter fairing and 55,000 kg payload to L2 eliminates this constraint (5).

\section{References}

1. Coulter, Daniel, R., “Technology development for the Next-Generation Space Telescope: an overview”, Proc. SPIE Int. Soc. Opt. Eng. Vol. 3356, page 106 (1998)

2. Stahl, H. Philip, “JWST mirror technology development results”, SPIE Proc.6671, 667102 (2007)

3. Stahl, H. Philip, “JWST Lightweight Mirror TRL-6 Results”, IEEE Aerospace Conference, March 2007.

4. Stahl, H. Philip, and Lee Feinberg, “Summary of NASA Advanced Telescope and Observatory Capability Roadmap”, IEEE Aerospace Conference, March 2007.

5. Stahl, H. Philip, “Ares V launch capability enables future space telescopes”, SPIE Proc.6687, 66870L (2007) 